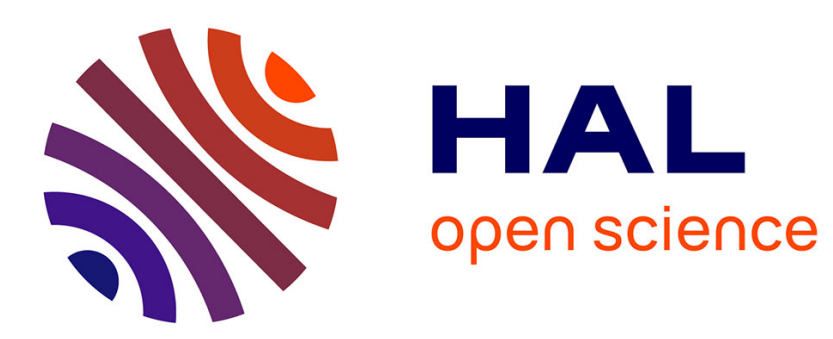

\title{
Réalités des échanges en Méditerranée orientale du XIIe au XVIIIe siècle: l'apport de la céramique
}

Véronique François

\section{To cite this version:}

Véronique François. Réalités des échanges en Méditerranée orientale du XIIe au XVIIIe siècle : l'apport de la céramique. Dumbarton Oaks Papers, 2004, 58, pp.241-249. 10.2307/3591387 • halshs-00751781

\section{HAL Id: halshs-00751781 https://shs.hal.science/halshs-00751781}

Submitted on 29 Jul 2020

HAL is a multi-disciplinary open access archive for the deposit and dissemination of scientific research documents, whether they are published or not. The documents may come from teaching and research institutions in France or abroad, or from public or private research centers.
L'archive ouverte pluridisciplinaire HAL, est destinée au dépôt et à la diffusion de documents scientifiques de niveau recherche, publiés ou non, émanant des établissements d'enseignement et de recherche français ou étrangers, des laboratoires publics ou privés. 
Réalités des échanges en Méditerranée orientale du XIIe au XVIIIe siècles: L'apport de la céramique

\section{Author(s): Véronique François}

Source: Dumbarton Oaks Papers, 2004, Vol. 58 (2004), pp. 241-249

Published by: Dumbarton Oaks, Trustees for Harvard University

Stable URL: http://www.jstor.com/stable/3591387

JSTOR is a not-for-profit service that helps scholars, researchers, and students discover, use, and build upon a wide range of content in a trusted digital archive. We use information technology and tools to increase productivity and facilitate new forms of scholarship. For more information about JSTOR, please contact support@jstor.org.

Your use of the JSTOR archive indicates your acceptance of the Terms \& Conditions of Use, available at https://about.jstor.org/terms 


\title{
Réalités des échanges en Méditerranée orientale du XIIe au XVIIIe siècles: l'apport de la céramique
}

\author{
VÉRONIQUE FrANÇOIS
}

$\mathrm{D}$

resser un bilan de la circulation de la vaisselle en Méditerranée orientale est une entreprise ambitieuse compte tenu du peu d'information dont nous bénéficions pour appréhender ce commerce et parce que les rapports et les équilibres dans le bassin méditerranéen au Moyen Age et au début de l'époque ottomane sont très changeants, que les marchés sont mouvants et que les retournements de conjoncture sont nombreux. Ce que nous savons, grâce aux découvertes archéologiques et, dans une moindre mesure, grâce aux textes, c'est que la vaisselle circule et parfois fort loin de ses centres de fabrication. Ce que nous ignorons souvent, ce sont les modalités de cette circulation. Grâce aux sources écrites, qui livrent des informations éparses mais précieuses, il est parfois possible d'appréhender les modes de diffusion de la céramique aux époques médiévale et ottomane. ${ }^{1}$ Ces textes, rédigés par des Occidentaux dans un contexte occidental ou oriental, sont de natures différentes: il s'agit d'actes de nolisement, de comptes de péages, de registres de taxation, d'inventaires mobiliers et d'inventaires après décès, de chroniques diverses et de récits de voyages ou de listes de cadeaux diplomatiques. Ces documents ne concernent pas toute la Méditerranée-pour l'Empire byzantin ou pour les sultanats islamiques par exemple, de telles sources existent sûrement mais la recherche dans ce domaine n'est pas développée à la différence de ce qui se passe en France, en Italie ou en Espagne. C'est conjointement que les sources écrites et les archives du sol permettent de hiérarchiser les approvisionnements en flux principaux et secondaires, et en diffusion marginale ce que je montrerai dans une première partie. L'examen de la distribution en Méditerranée orientale de deux productions médiévales occidentales et extrême-orientales particulièrement bien diffusées, prouvera, dans une seconde partie, que les données matérielles peuvent remettre en cause ce que nous connaissons de l'histoire économique de cette période.

\section{Modes de Circulation de la vaisselle en Méditerranée}

Circulation et distribution organisée sur de longues et moyennes distances

En Méditerranée, la vaisselle est une marchandise sujette à un commerce maritime de longues distances; sa présence sur les sites est alors le résultat d'une distribution organisée.

${ }^{1}$ Si j'ai choisi de faire déborder le cadre chronologique de cette étude au-delà du XVe siècle, c'est qu'il apparaît que la circulation de la céramique en Méditerranée n'est pas foncièrement différente avant et après la prise de Constantinople. 
Ce commerce à grande échelle s'exerce parfois par l'intermédiaire de réseaux internationaux. Ainsi la compagnie marchande et bancaire des Datini, en activité à Valence dès la fin du XIVe siècle, commande à plusieurs reprises de la vaisselle de terre à des potiers locaux pour le compte d'une compagnie de Florence-en décembre 1401, par exemple, ce sont 256 vases qui sont commandés à un potier de Manises (Fig. 1). ${ }^{2}$ Les sources écrites précisent que ces faïences sont chargées sur des navires dans le port de Valence, transitent par Palma de Majorque ou Ibiza, avant d'être livrées à Venise où il est difficile de savoir si elles sont uniquement destinées à une consommation locale ou si elles viennent aussi alimenter le commerce à destination de l'Orient.

Dans le cadre du commerce maritime, la poterie est un article de trop peu de prix pour constituer un chargement entier; le plus souvent, elle ne représente qu'un complément de cargaison. C'est aussi un produit d'appoint transporté d'une escale à une autre pour compléter, en fonction des ressources et des déficiences de chaque région, le cycle général des opérations marchandes. Diverses épaves ont livré des informations souvent incomplètes sur ce transport maritime. L'épave de Serçe Limanı datée du début du XIe siècle par des monnaies de Basile II et des poids en verre fatimides, retrouvée dans un port naturel de la côte turque méridionale, au nord de Rhodes, est parmi les plus intéressantes des épaves orientales car c'est la totalité de la cargaison qui est conservée. ${ }^{3}$ Nous pouvons donc estimer la place occupée par la vaisselle dans ce deux mâts équipé pour le cabotage et la remontée des rivières qui a probablement appareillé d'un port des côtes syro-palestiniennes à destination de la mer Noire. On trouve à bord des meules qui servent, comme c'est souvent le cas, de lest. Le ballast est aussi assuré par trois tonnes de verre brisé, une matière première destinée à la refonte dans les ateliers de verriers. Le navire transporte aussi du vin et des fruits secs dans une centaine d'amphores byzantines et divers objets manufacturés complètent le chargement: quatre-vingt vases en verre issus vraisemblablement d'ateliers syriens; six gargoulettes caractéristiques des productions fatimides d'Égypte; trois douzaines de céramique culinaire à pâte rouge et glaçure plombifère typiques des productions levantines; et quarante-quatre plats peints et incisés sous glaçure probablement d'origine syrienne. La vaisselle de table et de cuisine représente donc une infime quantité des produits transportés par ce navire à la différence d'un bateau naufragé au large de Pélagonnisos dans les Sporades du nord qui contenait dans ses cales environ mille cinq cents plats byzantins du XIIe siècle (Fig. 2, 3). ${ }^{4}$

Les comptes de péages et autres registres de taxation signalent également l'existence d'un commerce de gros de la poterie dans les bassins occidentaux et orientaux de la Méditerranée. Les comptes du péage de la ville d'Aigues-Mortes en Provence, en 1357, in-

${ }^{2}$ F. Melis, Documenti per la storia economica dei secoli XIII-XVI (Florence, 1972), 252, doc. 60.

${ }^{3}$ G. Bass, "The Shipwreck at Serçe Liman, Turkey," Archaeology 32.1 (1979): 36-43.

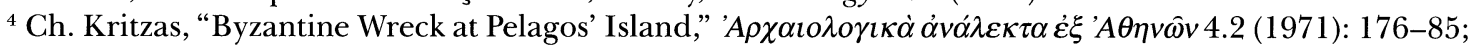
E. Ioannidaki-Dostoglou, "Les vases de l'épave byzantine de Pélagonnèse-Halonnèse," dans Recherches sur la céramique byzantine, BCH supplement 18, éd. V. Déroche et J.-M. Spieser (Athènes, 1989), 157-71. D’autres épaves contenant des chargements de vaisselle byzantine sont connues, mais elles ont livré moins d'informations sur ce transport: l'épave de Skopelos, dans les Sporades également, transportait des assiettes d'Aegean Ware de même que le navire retrouvé à Castellorizo, coulé au large des côtes lyciennes, sur la route qui joint Chypre et Rhodes à l'Égée: M. Michailidou et G. Philotheou, "Plats byzantins provenant d'une épave près de Castellorizo," ibid., 173-76; I. Loucas, "Les plats à glaçure inédits d'une collection privée de Bruxelles," ibid., $177-83$. 


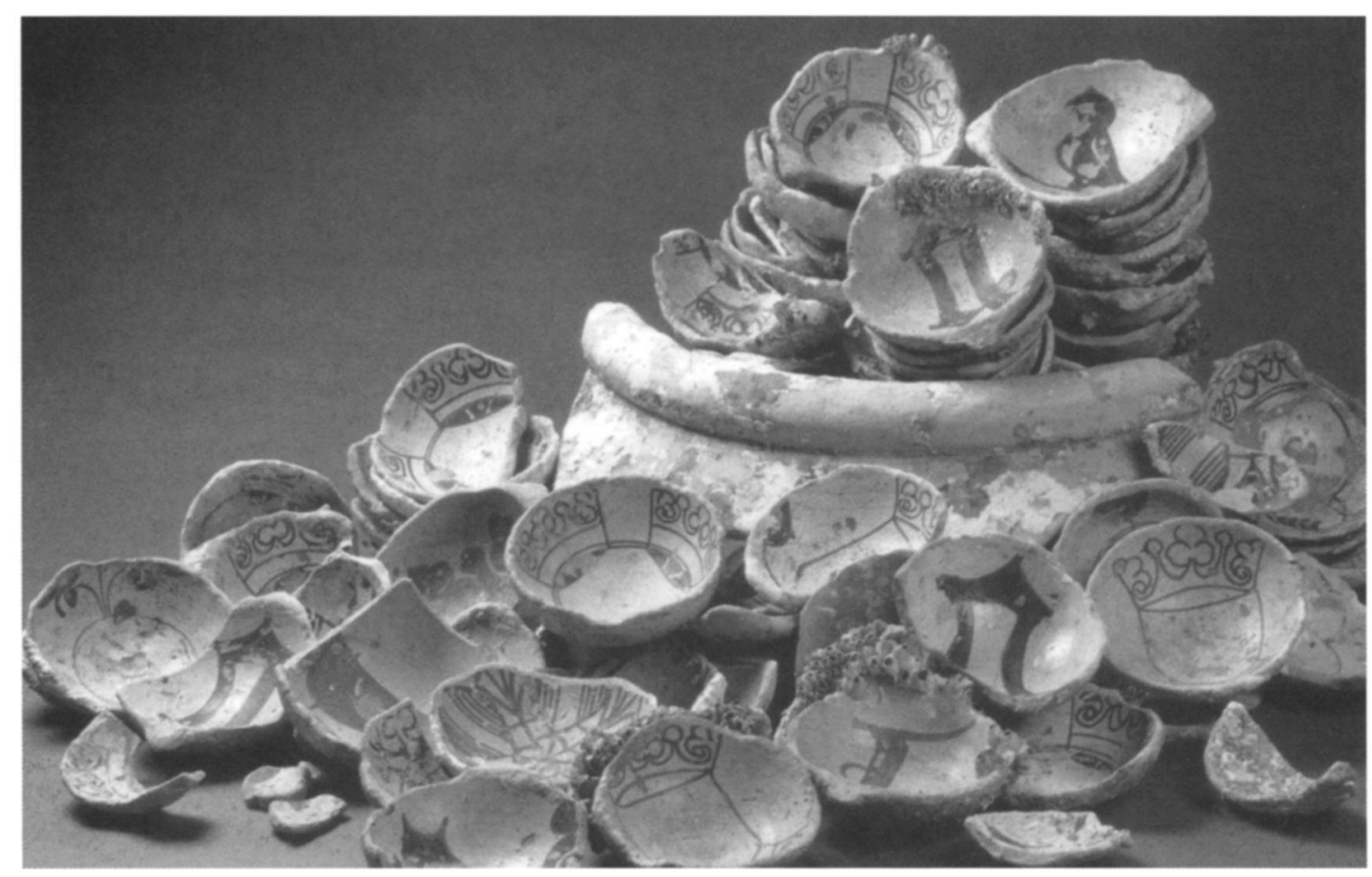

1 Faïences de Valence de l'épave de Carro (côtes provençales), milieu XVe s. (d'après H. Amouric, Fl. Richez, et L. Vallauri, Vingt mille pots sous les mers [Aix-en-Provence, 1999], 44, fig. 90; Sarl Edisud)

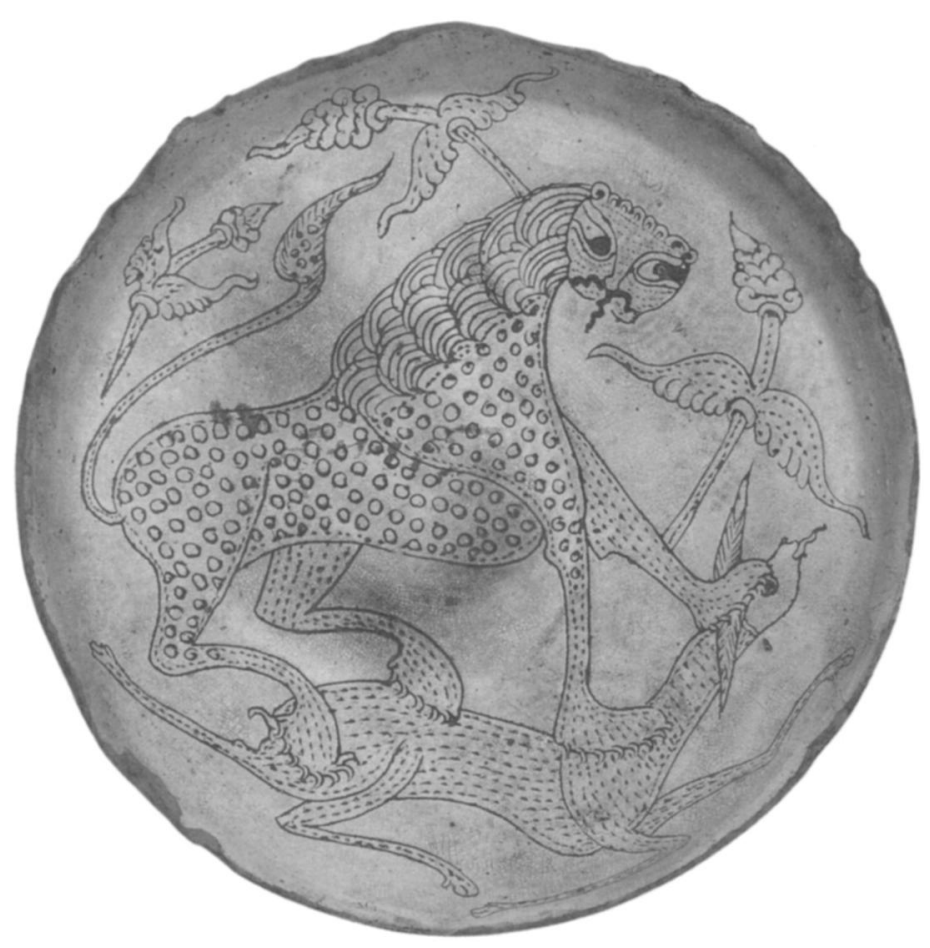

2 Fine sgraffito byzantin de l'épave de Pélagonnisos, milieu XIIe s. (d'après D. Papanikola-Bakirtzis, Byzantine Glazed Ceramics [Athens, 1999], 123, fig. 134; Archaeological Receipts Fund) 


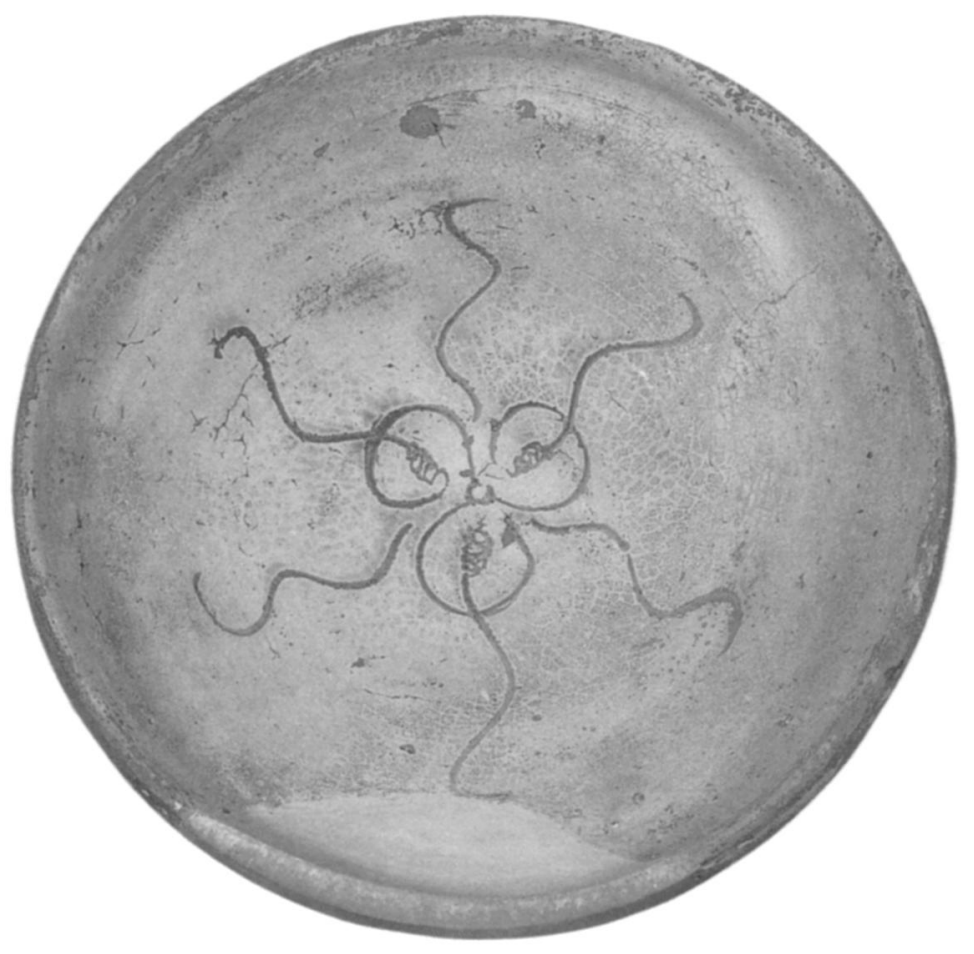

3 Aegean ware de l'épave de Castellorizo, début XIIIe s. (d'après D. Papanikola-Bakirtzis, Byzantine Glazed Ceramics, 150, fig. 175; Archaeological Receipts Fund)

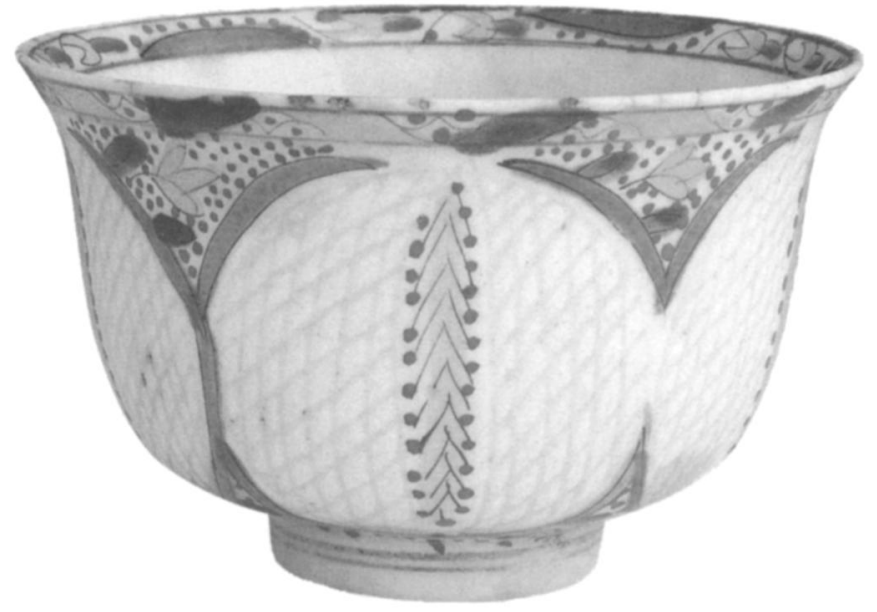

4 Fincan de Kütahya, première moitié XVIIIe s. (d'après L. Soustiel, Splendeurs de la céramique ottomane du XVIe au XIXe siècle [Istanbul, 2000], 116, fig. 64; Fondation Vebhi Koç) 


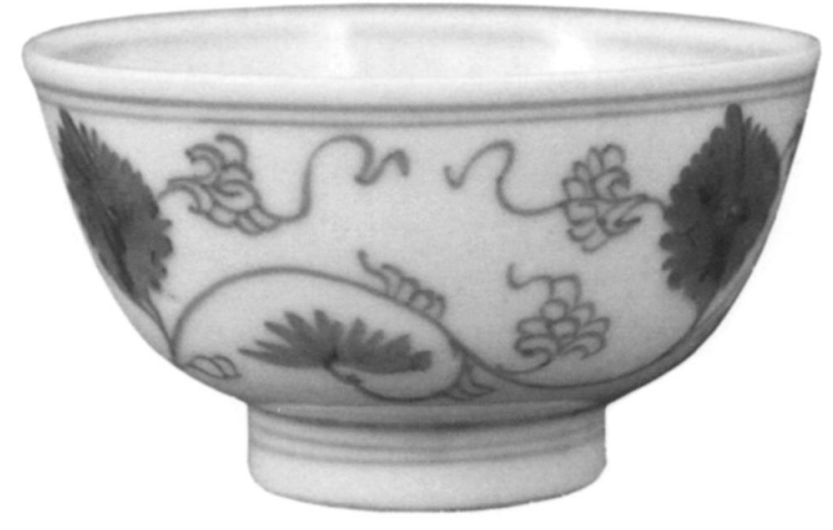

5 Tasse de porcelaine chinoise, fin XVIIe-début XVIIIe s. (d'après J. Carswell, Chinese Ceramics in the Sadberk Hanım Museum [Istanbul, 1995], 105, fig. 128; Fondation Vehbi Koç)

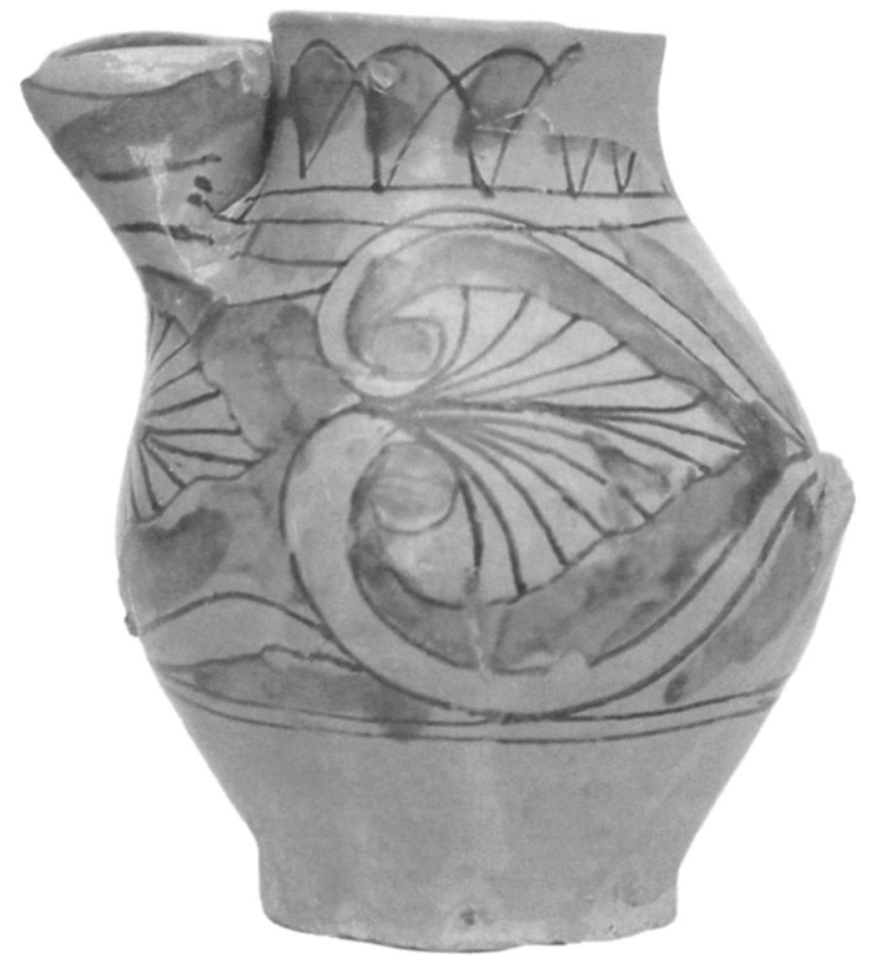

6 Cruche de proto-majolique, région d'Orvieto, XIIIe s. (d'après A. Ragona, La maiolica siciliana da le origini all'ottocento [Palermo, 1975], 43, fig. 17) 


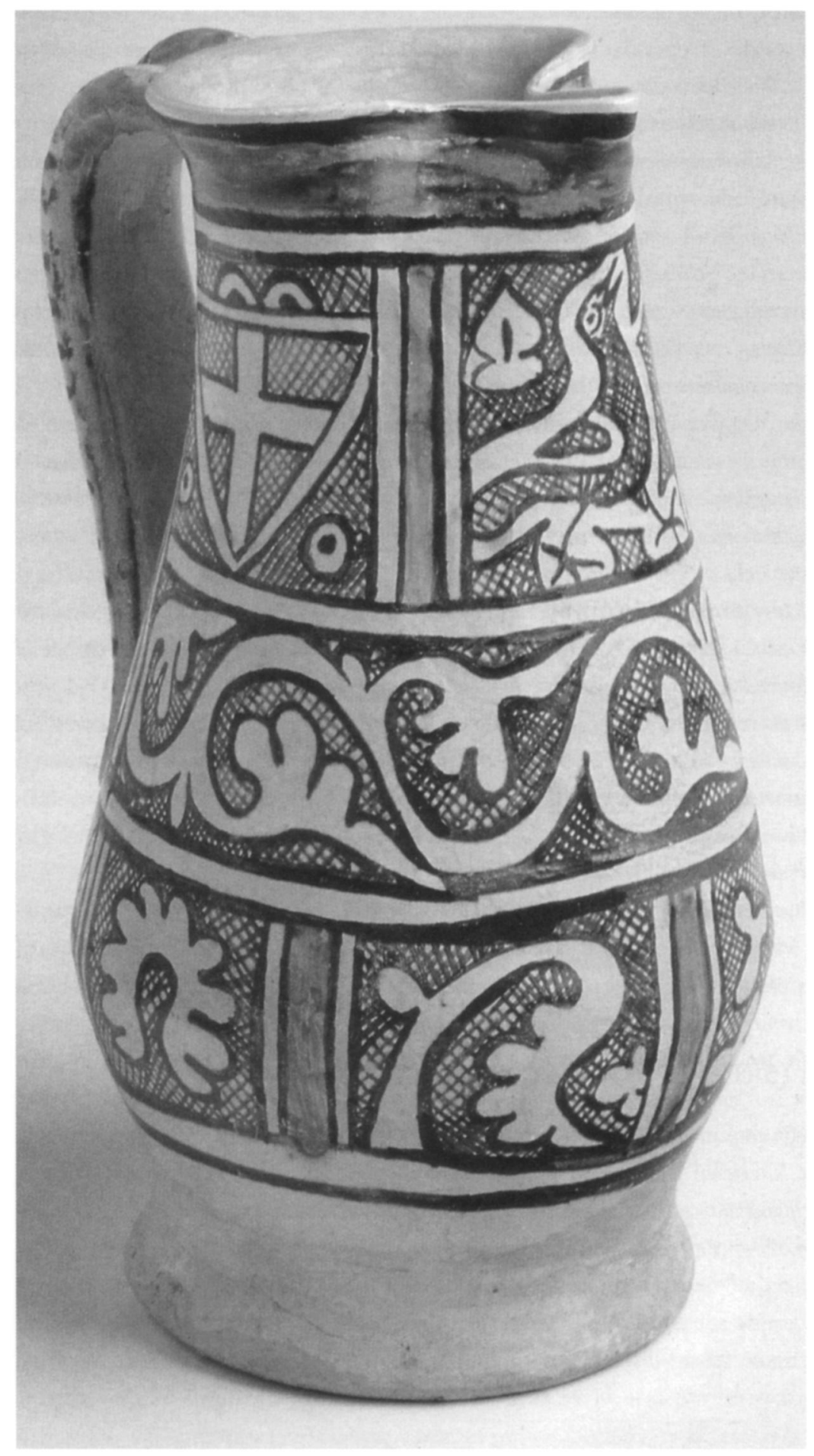

7 Cruche de majolique archaïque, XIVe s. (d'après S. Gelichi, "La ceramica a Faenza nel Trecento. Il contesto della Cassa Rurale a Artigiana," Faenza [1992], fig. 123) 


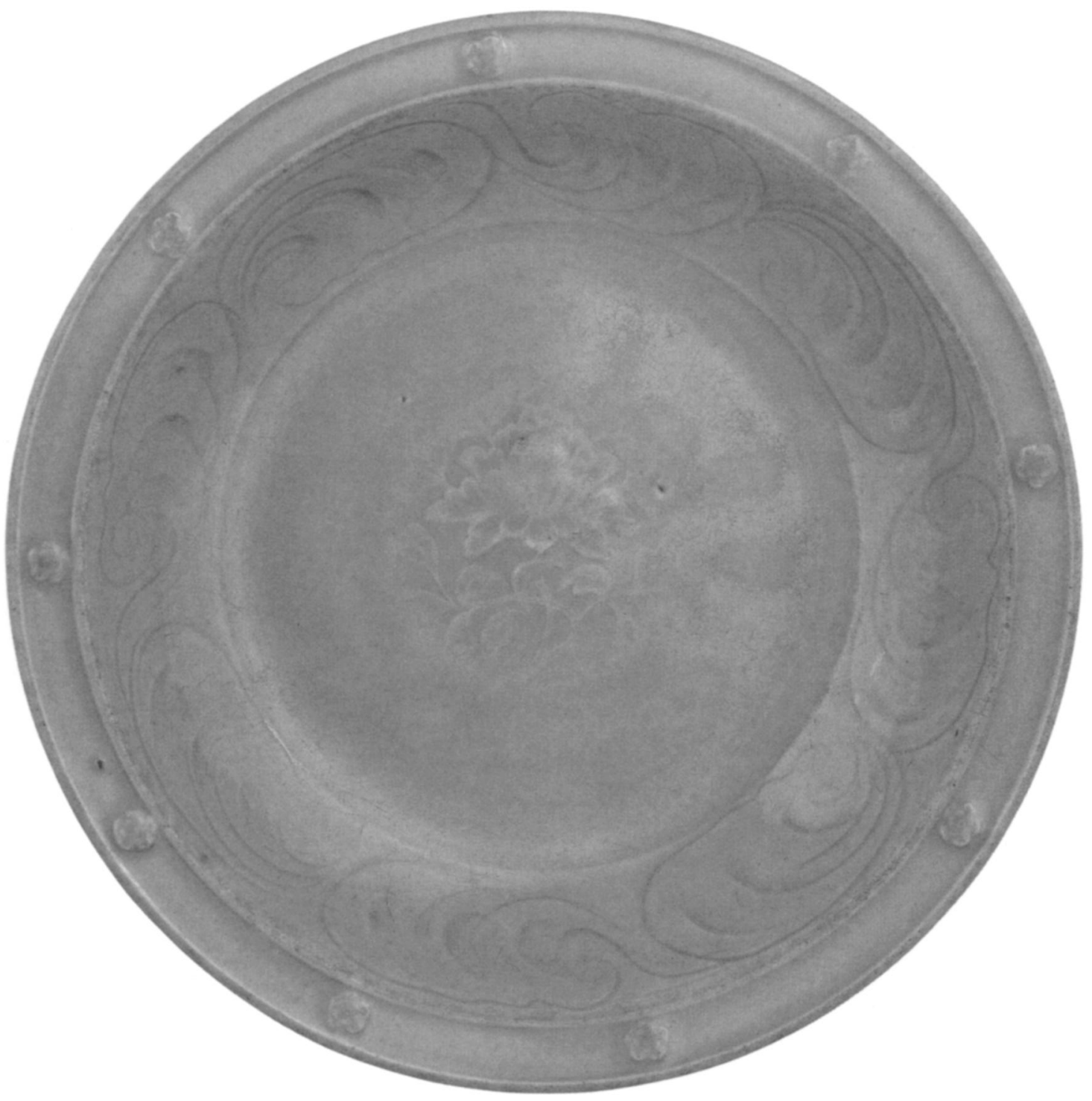

8 Céladon, première moitié XIVe s. (d'après Carswell, Chinese Ceramics in the Sadberk Hanım Museum, 25, fig. 7; Fondation Vehbi Koç)

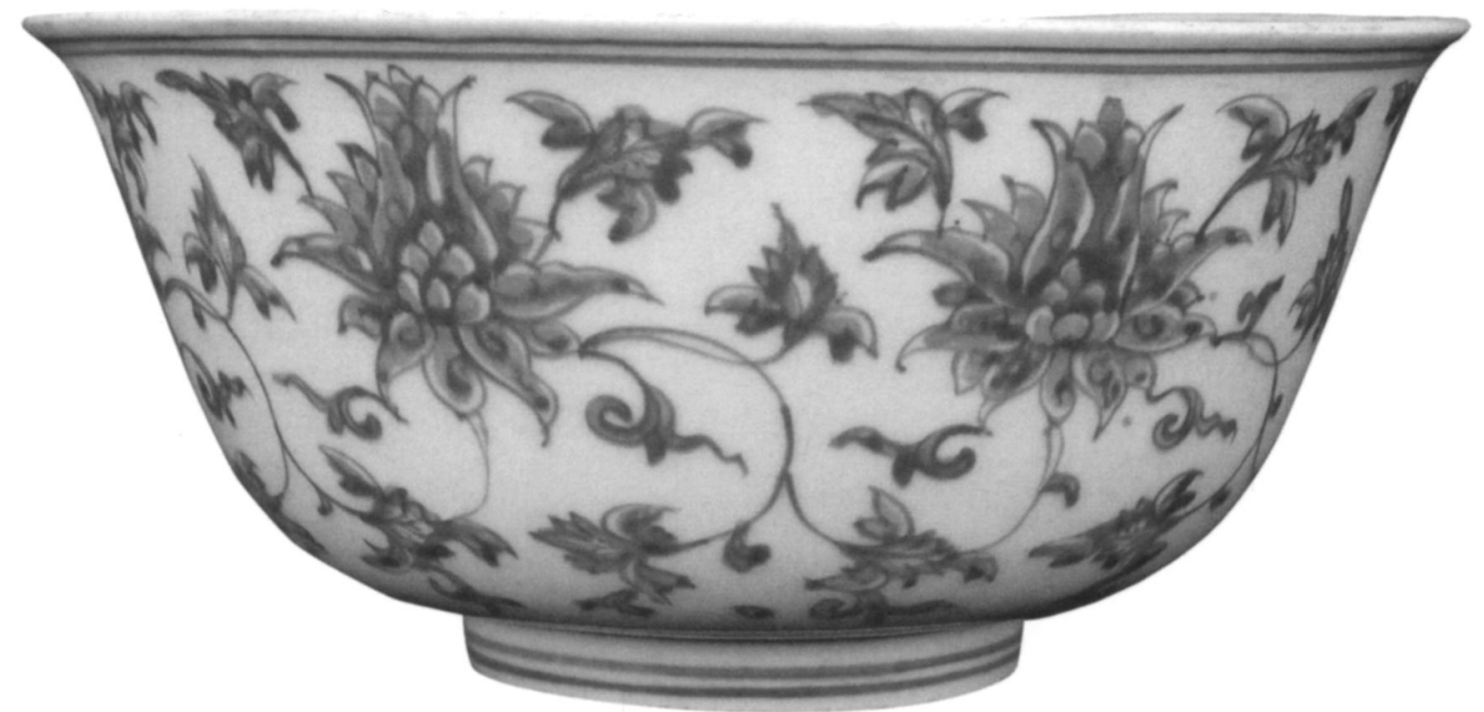

9 Porcelaine bleu-blanc, fin XVe-début XVIe (d'après Carswell, Chinese Ceramics in the Sadberk Hanım Museum, 52, fig. 44; Fondation Vehbi Koç) 
diquent dix-huit mentions de déchargement ou de passages en direction d'Arles ou de Marseille de céramiques fabriquées en Espagne et convoyées par barques; ${ }^{5}$ les tarifs du péage de Meyrargues, sur la Durance, font état en 1367 mais aussi en 1626 d'opus de Domasco-des pots, des écuelles et des pichets originaires de Damas-taxés par charge; ${ }^{6}$ au XIIIe siècle, les droitures de la fonde d'Acre précisent que les importations et les exportations de poterie sont soumises à taxation; ${ }^{7}$ à Beyrouth, parmi les privilèges accordés aux Génois en 1223, les labours de poterie sont exemptés de franchise commerciale; ${ }^{8}$ à Chypre, dans la première moitié du XIVe siècle, les scodelle di terra font partie de la longue liste de marchandises d'un prix relativement important et sujettes à la taxation qui sont importées d'Arménie, de Syrie, de Turquie et de Rhodes; ${ }^{9}$ enfin en 1457, le Grand Conseil de Raguse ordonne de prélever, en plus des taxes normales, un pour cent supplémentaire sur toutes les marchandises en provenance ou en partance pour Venise à l'exception des coupes, des tasses et des vases. ${ }^{10}$

L'examen attentif de la distribution de certaines productions permet d'appréhender aussi leurs modes de diffusion. Ainsi, D. Pringle, ${ }^{11}$ dressant l'inventaire des types les plus fréquemment rencontrés sur les sites de Syrie/Palestine, constate que là où la protomajolique italienne de la fin XIIe-XIIIe siècle est attestée lui sont presque toujours associées deux productions byzantines-Zeuxippus Ware et Aegean Ware-ainsi qu'une production levantine-St. Symeon Ware-en partie originaire des ateliers d'Al Mina, le port d'Antioche alors sous contrôle franc. ${ }^{12}$ A l'inverse, dans le Royaume latin de Jérusalem, à de rares exceptions, lorsque la proto-majolique est absente, les autres céramiques le sont aussi. Ces associations répétées de productions italiennes, byzantines et levantines constituent les indices d'un réseau de distribution commun-sans doute un commerce de cabotage alimenté par de grands centres qui servent de relais dans la diffusion de la vaisselle.

La distribution de la poterie suit parfois les réseaux commerciaux établis pour d'autres produits. Ainsi en Occident, par exemple, divers contrats indiquent que les faïences des ateliers de la région de Valence en Espagne bénéficient, aux XIVe-XVe siècles, du réseau de distribution du secteur textile-plusieurs marchands d'étoffe et des tailleurs de Narbonne, venus à Valence vendre leurs marchandises, repartent ensuite avec des chargements de vaisselle locale. ${ }^{13}$ Les céramiques circulent aussi en tant que contenants de

${ }^{5}$ H. Amouric, Fl. Richez et L. Vallauri, Vingt mille pots sous les mers (Aix-en-Provence, 1999), 32.

${ }^{6} \mathrm{~J}$. Thiriot, De l'Orient à la table du pape. Limportation des céramiques dans la région d'Avignon au Moyen Age tardif (XIVe-XVIe siècles), éd. D. Carru (Avignon, 1995), 27.

${ }^{7} \mathrm{~J}$. Richard, "Colonies marchandes privilégiées et marché seigneurial. La fonde d'Acre et ses droitures," $L e$ Moyen Age 59 (1953): 330-31; D. Jacoby, "The fonde of Crusader Acre and Its Tariff: Some New Considerations," dans Dei Gesta per Francos. Études sur les croisades dédiées à Jean Richard, éd. M. Balard, B. Z. Kedar et J. Riley-Smith (Aldershot, 2001), 284.

${ }^{8}$ Richard, "Colonies," 330 n. 11.

${ }^{9}$ L. Balletto, "Cipro nel 'Manuale di Mercatura' di Francesco Balducci Pegolotti," Miscellanea di studi storici 2, Collana storica di fonti studi 38 (1983): 138; Francesco Balducci Pegolotti, La Pratica della mercatura, éd. A. Evans (Cambridge, 1936), 86.

${ }^{10}$ B. Krekić, Dubrovnik (Raguse) et le Levant au Moyen Age (Paris, 1961), 403.

${ }^{11}$ D. Pringle, "Some More Protomaiolica from 'Atlit (Pilgrim's Castle) and a Discussion of Its Distribution in the Levant," Levant 14 (1982): 109.

${ }^{12}$ Sur l'existence d'autres ateliers voir notamment S. Redford, "Excavations at Medieval Kinet, Turkey: A Preliminary Report,” Ancient Near Eastern Studies 38 (2001): 58-138.

${ }^{13}$ P. Lopez Elum, "Origen y evolución de dos grandes centros ceramicos: Manises y Paterma," dans La ceramica nel Mediterraneo occidentale, Siena-Faenza, ottobre 1984 (Florence, 1986), 169. 
transport et de resserre; c'est alors ce qu'elles contiennent qui détermine leur diffusion. C'est le cas des albarelli, des pots tubulaires utilisés pour la conservation des épices naturelles ou de diverses préparations pharmacologiques. Dans le Sud de la France et en Italie, la plupart des albarelli recensés proviennent des ateliers espagnols de Valence en activité aux XIVe et XVe siècles et des centres de fabrication de Syrie de l'époque mamelouke. Ils ont servi d'emballage à des produits rares, et les textes révèlent fréquemment leur presence chez les apothicaires. ${ }^{14}$ De la même façon, à une époque plus récente, en 1559, une importante quantité de pots à pharmacie en majolique fabriqués à Savone en Ligurie est transportée jusqu'au couvent franciscain de St Sauveur à Jérusalem. ${ }^{15}$ C'est encore comme contenants que circulent, au départ de Montpellier vers Paris, des pots de terre vraisemblablement syriens qui contiennent de la confiture de Damas comme l'indique un acte notarial languedocien de $1409 .^{16}$

\section{Diffusion marginale de petits volumes}

Les usages maritimes ainsi que les aléas de la navigation sont aussi à l'origine du déplacement sur de longues distances et en petites quantités de certaines productions retrouvées loin de leurs centres de fabrication. Les usages maritimes occidentaux prescrivent en effet que chaque passager qu'il soit marchand ou pèlerin doit se munir de tous les objets nécessaires à son confort à bord. L'achat de vaisselle dans le port de partance est signalé dans plusieurs témoignages de la deuxième moitié du XIVe siècle: deux Italiens se rendant en Terre Sainte achètent à Venise avant l'embarquement, le 4 septembre 1384, un matelas, une bonne bouteille de Malvoisie, un petit coffre pour y ranger la Bible et les Évangiles ainsi que des tasses d'argent et des assiettes de terre ${ }^{17}$ un certain Giovanni Livi, se souvenant "di tutte quelle chose che si fanno bisogno per il mare," mentionne notamment trois grandes cruches, six assiettes, deux cuvettes et deux marmites à glaçure. Lors des aléas du voyage, pendant des tempêtes, par exemple, toute cette vaisselle embarquée peut se briser, les voyageurs se réapprovisionnent alors à l'occasion d'une escale. ${ }^{18}$ Ce système de distribution détournée est à même de justifier des découvertes isolées.

Certaines pratiques culturelles sont aussi à l'origine de découvertes en faible nombre de certains types de vases. C'est le cas des fincans, des tasses à café produites dans les ateliers ottomans d'Iznik et de Kütahya du XVIe au XVIIIe siècle (Fig. 4). Dans l'Empire ottoman, leur usage est très répandu, et on retrouve leur trace non seulement dans des contextes urbains mais aussi sur des sites ruraux et parfois dans des habitations relativement modestes. ${ }^{19}$ La place de la tasse à café dans la culture ottomane est à ce point importante

${ }^{14}$ Thiriot, De l'Orient à la table, 28; Amouric et al., Vingt mille pots, 40.

${ }^{15}$ D. Pringle, "Italian Pottery from Late Mamluk Jerusalem: Some Notes on Late and Post-Medieval Italian Tradewares in the Levant," Atti XVII Convegno Internazionale della Ceramica, Albisola, 25-27 maggio 1984 (Albisola, 1984), 40 .

${ }^{16}$ Amouric et al., Vingt mille pots, 115.

${ }^{17}$ C. Angelini, Viaggi in Terrasanta (Florence, 1944), 120.

${ }^{18}$ Le récit du voyage de Venise à Jérusalem du frère Niccolo da Poggibonsi, en 1346, relate que durant une tempête "tutti gli storigli si romperano": F. Zambrini, Viaggio da Venezia a Gerusalemme di Fr. Niccolo da Poggibonsi (Bologne, 1872), 56.

${ }^{19}$ J. Vroom, "Coffee and Archaeology. A Note on a Kütaya Ware Find in Boeotia, Greece," Pharos 4 (1996): 5-17; V. François, "Éléments pour l'histoire ottomane d'Aphrodisias: La vaisselle de terre," Anatolia Antiqua. Eski Anadolu (Istanbul) 9 (2001): 147-90. 
qu'elle est considérée comme l'équipement ordinaire du nécessaire à café du parfait caravanier qui comporte un sac de cuir pour mettre le café, un petit coquard pour le faire cuire, et une boîte cylindrique en cuivre ou un étuis en maroquin pour y ranger les tasses, ${ }^{20}$ ce qui permet un transport sans risque jusqu'au moment où le fincan se brise et qu'on l'abandonne sur place. Cette pratique peut expliquer la large dispersion de ces objets mais en très faible volume.

D'autres découvertes sporadiques révèlent une ouverture au monde et à l'exotisme. A l'époque ottomane, il n'est pas rare que les pèlerins lors de leur séjour à La Mecque achètent quelques tasses en porcelaine de Chine (Fig. 5) comme objets souvenir ainsi que le rélèvent les inventaires après décès de certains de ces voyageurs morts à Damas. ${ }^{21}$ Ces porcelaines fabriquées dans les ateliers chinois sont même à l'occasion désignées de Kabe fincani-autrement dit tasse à café de la $\mathrm{Ka}$ 'ba-comme on peut le lire dans l'inventaire de la boutique d'un marchand d'Ankara au XVIIe siècle. ${ }^{22}$

Faïences et porcelaines sont également l'objet de prise de guerre. Ainsi l'introduction des vases de Chine dans la capitale de l'Empire ottoman est la conséquence des conquêtes de Selîm Ier. Lors de la prise de Tabriz en 1514, il récupère un butin de soixante-quatre plats chinois; puis en 1516-17, il enrichit de nouveau le trésor impérial après les prises de Damas et du Caire où il s'empare de la riche collection du sultan mamelouk Qânsûh alGhûrî. ${ }^{23}$ La vaisselle de luxe est aussi l'objet de cadeaux qui contribuent à honorer les puissants quelles que soient les périodes. Ainsi, dès 804, le gouverneur du Korasan offre au calife Hârûn al-Rashîd vingt vases de céramique chinoise de qualité et deux cents vases plus communs; en 1171, quarante vases chinois sont remis par Salâh al-Dîn au prince Nûr al-Dîn de Damas; et deux siècles plus tard, en 1392, ce sont cinq cents porcelaines qui sont déposées aux pieds du sultan rasulid du Yémen à l'occasion de la circoncision de son fils. ${ }^{24}$ Les archives européennes livrent aussi des listes de présents diplomatiques échangés entre souverains: lors de pourparlers commerciaux entre la France et l'Égypte, en 1447, Charles VII reçoit des porcelaines de la part du sultan mamelouk; de la même façon Laurent de Médicis est gratifié par le sultan Qaytbay de vases de porcelaine lors de la signature d'accord de commerce entre la République de Toscane et l'Égypte; en 1476, ce sont quatorze porcelaines qui sont offertes par le sultan à Catherine Cornaro à Chypre; enfin de 1442 à 1503, nombreux sont les doges de Venise à recevoir de la part du pouvoir mamelouk diverses faïences et porcelaines. ${ }^{25}$

Dans certaines circonstances, seuls les textes sont à même d'expliquer des découvertes rares et montrent combien les voies de pénétration de la vaisselle peuvent être détournées,

${ }^{20}$ H. Desmet-Grégoire, Les objets du café (Paris, 1989), fig. 78; eadem, “Origine et évolution du café à Marseille aux XVIIe et XVIIIe siècles," Provence historique 38, fasc. 151 (1988): 78; Amouric et al., Vingt mille pots, 161.

${ }^{21} \mathrm{C}$. Establet et J. P. Pascual, Ultime voyage pour La Mecque. Les inventaires après décès de pèlerins morts à Damas vers 1700 (Damas, 1998), 150-51.

${ }^{22}$ S. Faroqhi, Men of Modest Substance. House Owners and House Property in Seventeenth-Century Ankara and Kayseri (Cambridge, 1987), 28.

${ }^{23}$ J. Raby et Ü. Yücel, "Blue-and-White, Celadon and Whitewares: Iznik's Debt to China," Oriental Art 29 (1983): 39.

${ }^{24}$ A. Lane et B. Serjeant, "Pottery and Glass Fragments from the Aden Littoral, with Historical Notes," JRAS (1948): 110; P. Kahle, "Chinese Porcelain in the Lands of Islam," Transactions of the Oriental Ceramics Society (Oxford) (1940-41): 44 n. 33.

${ }^{25}$ M. Milwright, "Pottery in the Written Sources of the Ayyubid-Mamluk Period (c. 567-923/1171-1517)," BSOAS 62 (1999): 516. 
comme c'est le cas dans un document de 1475 dans lequel un certain Manualdus Guerra reconnaît devoir une certaine quantité de plats et d'écuelles de terre de Valence à deux Marseillais pour solde d'un loyer de maison. ${ }^{26}$

\section{DES SURPRISES DANS L'INTERPRÉTATION ÉCONOMIQUE ET CULTURELLE DE LA DISTRIBUTION DE LA VAISSELLE DE TERRE}

Il ne s'agit pas ici de dresser un inventaire des productions nombreuses et variées qui circulent en Méditerranée orientale au Moyen Age et au début de l'époque ottomane, ${ }^{27}$ mais plutôt d'insister sur les différences d'approvisionnement relevées sur des sites de régions voisines et qui sont des révélateurs de particularismes économiques ou culturels. L'examen de la diffusion des céramiques italiennes et des porcelaines chinoises bien représentées en Méditerranée orientale et qui, par leur fréquence d'apparition et le volume de leurs découvertes, sont symptomatiques de la réalité des échanges, constitue de ce point de vue un bon exemple.

\section{Absence de production médiévale italienne à Damas et à Chypre}

En Méditerranée orientale, du XIIIe au XVe siècle, les productions italiennes sont très présentes-on en trouve la trace dans les fouilles ainsi que sur les édifices religieux où elles sont employées comme décoration architecturale. ${ }^{28}$ Ce qu'on rencontre en majorité, ce sont des exemplaires de céramiques peintes sur émail—de la majolique archaïque (Fig. 7) et de la proto-majolique (Fig. 6) fabriquées dans le centre et le Nord de l'Italie pour l'une et dans le Sud de la péninsule et en Sicile pour l'autre-et diverses céramiques à glaçure au plomb originaires de Venise et de sa région. ${ }^{29}$ Dans l'Empire byzantin, la présence de ces importations italiennes apparaît à l'évidence comme la conséquence d'un système de transport et de distribution favorable à une telle diffusion dans le cadre de contacts politiques et économiques multipliés entre l'Italie et la Grèce. Les principales attestations de ces poteries concernent une vingtaine de sites qui ont tous en commun d'avoir été directement sous contrôle franc ou d'avoir entretenu d'étroites relations politiques ou économiques avec l'Italie. ${ }^{30}$ Au Levant, la plupart des sites tombés aux mains des Croisés ont livré des importations de mêmes types liées également à l'occupation latine et au commerce maritime italien. ${ }^{31}$ Sur les sites islamiques de Méditerranée comme Alexandrieune plaque tournante pour les échanges entre l'Orient et l'Occident-la variété des pro-

\footnotetext{
${ }^{26}$ Amouric et al., Vingt mille pots, 40.

${ }^{27}$ À noter qu'il n’y a pas que la vaisselle de table et de service haut de gamme qui a été objet de commerce; la céramique culinaire et des productions glaçurées rustiques ont également bénéficié d'une large distribution.

${ }^{28}$ Pour un inventaire détaillé des découvertes en Grèce et en Turquie voir V. François, "Céramiques importées à Byzance: Une quasi absence,” BSl 58 (1998): 387-404; pour le Levant, se reporter notamment à Pringle, "Some More Protomaiolica," 104-17; idem, "Pottery as Evidence for Trade in the Crusader States," dans I comuni italiani nel regno latino di Gerusalemme (Gênes, 1986), 451-75.

${ }^{29}$ Sur ces différents types voir François, "Céramiques importées," 392-95.

${ }^{30}$ Ibid., 395-400.

${ }^{31}$ Pringle, "Pottery as Evidence," 451-75; S. Gelichi, "La ceramica bizantina in Italia e la ceramica italiana nel Mediterraneo orientale tra XII e XIII secolo: Stato degli studi e propose di ricerca," dans La ceramica nel mondo bizantino tra XI e XV secolo e i suoi rapporti con l'Italia, Atti del Seminario, Certosa di Pontignano (Siena), 11-13 marzo 1991 (Florence, 1993), 9-46.
} 
ductions italiennes mises au jour est quasiment identique. ${ }^{32}$ Cependant, Damas et Chypre, deux régions du Proche-Orient pourtant considérées elles aussi comme d'importants relais du commerce international au Moyen Age, se distinguent dans leur approvisionnement en vaisselle italienne.

Dans les fouilles de la citadelle de Damas, ${ }^{33}$ les productions italiennes d'époque médiévale sont absentes à la différence de ce qu'on trouve sur les sites côtiers de Syrie/Palestine. Proto-majoliques, majoliques et graffita n'ont pas atteint cette ville de l'intérieur. Certes le commerce syrien, entre la fin du XIIIe et la fin du XVe siècle, traverse une mauvaise conjoncture-Damas se trouve à l'écart des grandes voies d'échanges avec l'Occident à cause du déplacement des routes commerciales vers le Nord, c'est-à-dire vers la Mer Noire, par la Crimée ou Trébizonde, de 1240 à 1340; de plus l'édit pontifical qui interdit aux états chrétiens de commercer avec les Mamelouks contraint Alep et Damas à céder une partie considérable de leur trafic aux villes portuaires du Royaume chrétien de Petite Arménie. ${ }^{34}$ Mais si Damas est considérée, du point de vue de l'histoire économique, comme une grande échelle de la Méditerranée orientale tout autant qu'Alexandrie ou Constantinople, les découvertes de céramiques ne vont pas dans ce sens. D'un point de vue céramologique, Damas, durant l'époque médiévale, n'est pas une ville méditerranéenne et son approvisionnement en vaisselle ressortit à d'autres réseaux commerciaux. La vaisselle italienne apparaît seulement dans la ville à l'époque ottomane. Les fouilles de la citadelle ont livré quelques productions de la Renaissance réalisées, aux XVIe et XVIIe siècles, dans des ateliers d'Italie du nord et de Toscane.$^{35}$ Leur faible nombre exclu toutefois une commercialisation à grande échelle. ${ }^{36}$

En l'état des découvertes et sur la base du matériel publié, on constate que, curieusement, l'approvisionnement de Chypre en céramique italienne semble calqué sur celui de Damas. En effet, sur l'île, excepté quelques proto-majoliques découvertes à Famagouste et au château de Saranda Kolones à Paphos, les productions venues d'Italie au Moyen Age sont quasiment inexistantes alors que celles datées du XVe au XVIIIe siècle sont fréquentes. ${ }^{37}$ Ces vases, contemporains des découvertes damascènes, proviennent également d'Italie du nord et de Toscane. ${ }^{38}$ Cette situation est pour le moins étonnante car Chypre

${ }^{32}$ V. François, La céramique médiévale à Alexandrie. Contribution à l'histoire économique de la ville, Études Alexandrines 2 (Le Caire, 1999), 71-81.

${ }^{33}$ Ce matériel a été mis au jour lors des fouilles franco-syriennes dirigées par E. Ajji (Direction générale des Antiquités et des Musées de Syrie) et $\mathrm{S}$. Berthier (chercheur à l'Institut français d'études arabes de Damas). Son étude m'est confiée.

${ }^{34}$ E. Wirth, "Alep et les courants commerciaux entre l'Europe et l'Asie du XIIe au XVIe siècle," dans Villes au Levant. Hommage à André Raymond, RE.M.M.M. 55-56 (1990): 44-56.

${ }^{35}$ Pour le détail des types voir V. François, "Production et consommation de vaisselle à Damas à l'époque ottomane," BEODam 53-54 (2002): 157-70.

${ }^{36}$ Cette présence est par ailleurs faiblement attestée sur d'autres sites du Levant tels que Antioche et AlMina, Acre, Jérusalem et Nazareth: C. N. Johns, "The Citadel, Jerusalem. A Summary Work since 1934," QDAP 14 (1950): 189, pl. LXIII, n 5-7; Pringle, "Italian Pottery," 37-44; G. Edelstein et M. Avissar, "A Sounding in Old Acre," 'Atiqot 31 (1997): 132.

${ }^{37}$ La présence accrue de céramiques italiennes à Chypre à partir du XVe siècle est sans doute à mettre en rapport avec la prise de contrôle de Famagouste par les Génois de 1373 à 1464. Ensuite l'île passe sous protectorat vénitien avant d'être annexée par Venise en 1489.

${ }^{38}$ Pour un inventaire de ces types voir V. François et L. Vallauri, "Production et consommation de céramiques à Potamia (Chypre) de l'époque franque à l'époque ottomane,” BCH 125 (2002): 537-39, 545. 
est de facto un grand centre du commerce maritime de la Méditerranée européenne et la fréquentation de l'île par les marines latines est encore renforcée en 1291 par la chute d'Acre. On s'attend donc à y trouver le même type de matériel que celui mis au jour sur les sites côtiers de Syrie/Palestine. Mais l'île présente une particularité dans sa consommation de vaisselle; les vestiges matériels indiquent qu'elle a bénéficié d'autres modes de distribution que les sites francs du Levant et que les villes passées sous contrôle latin en Grèce.

\section{Absence de porcelaine chinoise à Byzance}

La seconde production dont il est intéressant d'examiner la diffusion est la céramique chinoise qui constitue la manifestation la plus visible des réseaux commerciaux qui, entre la IXe et le XVe siècle, relient la Chine au Moyen-Orient. En Iran, en Irak, en Syrie, au Liban, en Égypte et sur les côtes de la péninsule Arabique tant sur la Mer Rouge que dans le Golfe Persique, les fouilles ont livré des centaines, voire des milliers, de fragments de céramiques chinoises. Leur présence est d'abord attestée dans les villes portuaires du golfe Persique et dans les centres servant d'entrepôts le long des itinéraires commerciaux tels que Basra, Siraf et Sohar, ${ }^{39}$ ou encore à Bagdad $^{40}$ ainsi que dans de grandes cités comme Samarra, Nishapur, Suse ou Rey. ${ }^{41}$ Dès la fin du XIe siècle, la céramique est la première marchandise chinoise à l'exportation. C'est alors que les circuits du commerce avec le Moyen-Orient changent. Les navires délaissent progressivement le Golfe pour faire voile vers la Mer Rouge avec pour entrepôt principal Aden et comme relais du commerce oriental, suivant les périodes, les ports égyptiens d'Aydhab, de Tôr et dans une moindre mesure de Quseir al-Qadim. ${ }^{42}$ Dans tous ces lieux de débarquement égyptiens la céramique chinoise est présente de même qu'elle apparaît en quantité sur les grands marchés du Caire et d'Alexandrie. ${ }^{43}$

L'étude de la diffusion au Proche-Orient des porcelaines et des céladons nous renseigne sur les réseaux commerciaux mais aussi sur certains particularismes culturels. En effet, ce qu'on remarque c'est que ces importations chinoises concernent presque uniquement des sites islamiques. Dans les villes côtières de Syrie/Palestine occupées par les Latins

\footnotetext{
${ }^{39}$ J. B. da Silva, "Some Chinese Porcelain Found in South Arabia," Oriental Art 14.1 (1968): 41-45; A. Rougeulle, "Les importations de céramiques chinoises dans le golfe Arabo-Persique (VIIIe-XIe siècles)," Archéologie islamique (Paris) 2 (1991): 5-44; D. Whitehouse, "Some Chinese and Islamic Pottery from Siraf," in W. Watson, ed., Pottery and Metalwork in Tang China, Colloquies on Art and Archaeology in Asia 1 (London, 1976), 30-34; idem, "Chinese Stoneware from Siraf: The Earliest Finds," South Asian Archaeology (1973): 241-55; M. Kervran, A. Nègre et M. Pirazzoli T’Sertsevens, Fouilles à Qa'lat Al-Bahrein, 1er partie (1977-1979) (Bahrein, 1982), 52.

${ }^{40} \mathrm{G}$. Wiet, "Les marchands d'épices sous les sultans mamelouks," Cahiers d'histoire égyptienne (Le Caire) 7 (1955): 82.

${ }^{41}$ Lane et Serjeant, "Pottery and Glass Fragments," 109-13; M. Kervran, "Les niveaux islamiques du secteur oriental du tépé de l'Apadana. II. Le matériel céramique," Cahiers de la Délégation Archéologique Française en Iran 7 (1977): 92.

${ }^{42}$ Wiet, "Les marchands," 84; R. L. Hobson, "Chinese Porcelain from Aidhab, and Some Bashpa Inscriptions," Transactions of the Oriental Ceramics Society (Oxford) (1926-27): 19-22; D. S. Whitcomb, Quseir al-Qadim 1978, Preliminary Report (Le Caire-Princeton, 1979), 108-9, pl. 49d; T. Mikami, "Chinese Ceramics from Medieval Sites in Egypt," dans Bulletin of the Middle Eastern Culture Center in Japan, vol. II: Cultural and Economic Relations between East and West Sea Routes, éd. T. Mikasa (Wiesbaden, 1988), 13.

${ }^{43}$ Mikami, "Chinese Ceramics," 8-44; François, La céramique médiévale, 143-53.
} 
telles que Acre, Atlit, ou Césarée ou dans des installations franques comme le monastère de Sainte-Marie-du-Carmel à Haifa ${ }^{44}$-des sites tous remarquables pour leur approvisionnement varié en productions étrangères-céladons et porcelaines sont totalement absents alors qu'on trouve leur trace dans des villes sous domination mamelouke comme Ascalon, Hama, Antioche et Tripoli. ${ }^{45}$ On observe un phénomène identique en Anatolie où la vaisselle chinoise n'atteint pas les régions restées sous contrôle byzantin alors qu'elle est attestée sur les sites passés aux Seldjoukides et aux tribus turcomanes. ${ }^{46}$ Dans les fouilles ouvertes en Grèce et en Turquie, la céramique de Chine n'apparaît jamais dans les niveaux byzantins pourtant contemporains des découvertes moyen-orientales en contexte musulman. Byzance semble tout ignorer de ces vases de luxe ce qui est d'autant plus troublant que certaines conditions sont favorables à leur introduction et à leur commercialisation dans l'Empire; c'est-à-dire des moyens financiers suffisants permettant d'acquérir de la vaisselle de prix et l'existence de diverses routes commerciales convergeant à Constantinople qui auraient permis un tel approvisionnement si la demande avait existé. Le désintérêt manifesté en contexte chrétien pour cette vaisselle exotique exprime sans doute une différence culturelle majeure entre les classes sociales aisées musulmanes et chrétiennes.

Témoins épars et continus d'échanges proches ou lointains organisés ou non, poteries, faïences et porcelaines constituent la preuve de contacts établis entre des mondes parfois fort lointains et des cultures dissemblables. Afin de saisir les différences de consommation de la vaisselle de terre, sur la base des trouvailles archéologiques, des cartes de diffusion des types assortis au volume de leurs découvertes peuvent être établies. La circulation de ces poteries doit ensuite être envisagée dans le cadre des courants commerciaux connus par les recherches menées dans le domaine de l'histoire économique. Mais il arrive que des vases se trouvent là où on ne les attend pas ou au contraire fassent défaut là où on les espère. L'archéologue, à travers la présence ou l'absence de ces objets, pose alors des questions nouvelles aux historiens.

CNRS-Institut Français du Proche-Orient Centre National de la Recherche Scientifique

\footnotetext{
${ }^{44}$ D. Pringle, "Excavations in Acre, 1974: The Pottery of the Crusader Period from Site D," Atiqot 31 (1997): 137-56; idem, "Medieval Pottery from Caesarea: The Crusader Period," Levant 17 (1985): 171-202; idem, "Thirteenth-Century Pottery from the Monastery of Saint Mary of Carmel," Levant 16 (1984): 91-111.

${ }^{45}$ J. D. Frierman, "Chinese Ceramics from Ashkelon and Caesarea," IEJ 19 (1969): 44-46; P. J. Riis et V. Poulsen, Hama. Fouilles et recherches 1931-1938. Les verreries et poteries médiévales (Copenhague, 1957), 117-19; F. Waagé, Ceramics and Islamic Coins: The Glazed Pottery, Antioch on the Orontes 4.1 (1948), 104-5, figs. 93-94; H. Salamé-Sarkis, Contribution à l'histoire de Tripoli et de sa région à l'époque des Croisades: Problèmes d'histoire, d'architecture et de céramique, Bibliothèque archéologique et historique 106 (Paris, 1980), 225-26.

${ }^{46}$ V. François, "L'arrivée de l'Islam en Anatolie, un vecteur de diffusion de la céramique chinoise," Annales islamologiques (Le Caire) 32 (1998): 41-47.
} 